\title{
Stabilization of constrained uncertain systems by an off-line approach using zonotopes
}

\author{
Walid Hamd: Wissal Bey, Naceur Benhadj Braiek \\ Laboratory of Advanced Systems (L.A.S), Polytechnic School of Tunisia, Carthage University, BP 743,2078 La \\ Marsa, Tunisia
}

A R T I C L E I N F O

Article history:

Received: 30 November 2017

Accepted: 07 January 2018

Online: 31 January 2018

Keywords:

Stabilization

Zonotopic invariant sets

Model Predictive Control

Uncertain systems

\section{Introduction}

Model predictive control (MPC) is one of the most successful techniques of advanced control in the process industry. Thanks to the recent developments of the underlying theoretical framework, MPC has become a mature control technique able to provide controllers ensuring stability, robustness, constraint satisfaction and tractable computation for linear and nonlinear systems [1]. The MPC is can be made in the context of representation in state variables [2]. This not only make use of existing theorems and results in the state space theory, but also facilitates the extension of the theory of model predictive control to more complex cases such as systems with stochastic disturbances, noise on measured variables or multivariable control. For nonlinear uncertain systems, explicitly modeling of the uncertainty is essential [3].

For modeling uncertain systems, it is very important for MPC to be more robust [2]. Important areas in MPC that have recently seen significant theoretical and implementational progress include robust and stochastic MPC as well as efficient computations for MPC via convex and reliable real-time optimization [4].

Although these MPC schemes have remarkable performance and good theoretical properties, there is a hard computational burden due to the minmaximization of the optimization problem, especially in the presence of the system nonlinearity. The other is to derive robust stability of MPC by minimization of linear quadratic optimization problems subject to polytopic uncertainty models and linear matrix inequality (LMI) constraints, which was firstly proposed in [5]. From this formulation, a broad class of model uncertainty descriptions can be addressed with guaranteed closed-loop robust stability of MPC.

Since the Lyapunov theory was introduced as an efficient stability analysis tool of systems governed by ordinary differential equations, the notion of set invariant was used in many problems concerning the analysis and control of dynamic systems. An important motivation, leading to introduce invariant sets, was the need to analyze the effect of uncertain systems. An invariant set is a region of the state space such the trajectory generated by the dynamical system remains confined in the set if the initial condition lies within it [6]. Robust controlled invariant set is particularly relevant since it can be used in the context of constrained uncertain systems stability [7].

In recent years, in the theory of control, regardless of a particular area, there have been numerical solutions are extensive. That is, a problem is usually considered as solved whenever it can be written as a (constrained) optimization problem. The difficulty in solving such a problem is greatly influenced by the way the constraint set is defined. In this context, several families of sets vie for influence [8].

Historically, ellipsoidal sets [9] were a useful choice of invariant sets due to their simple definition.

\footnotetext{
${ }^{*}$ Corresponding Author: hamdi.walid987@gmail.com 
Then, the problem becomes to design the invariant ellipsoids off-line [10]. Recently, polyhedral sets [11], became widespread due to their representation flexibility and reliable numerical algorithmes. Angeli [12] proposed an ellipsoidal off-line MPC scheme for uncertain polytopic systems. In [13] the authors proposed an off-line robust constrained MPC algorithm by choosing a sequence of states.

However, polyhedral sets become numerically unstable for higher dimensions and certain operations scale badly with respect to the complexity of the set in question. Zonotopic sets, a subclass of polyhedral sets [11], have started to gain attention. Their symmetric shape, coupled with the flexibility inherited from the polyhedral class makes them an appealing choice for higher dimensions. Also, for dynamical systems, zonotopes provide an excellent compromise between accuracy and efficiency as first [14]. As a direct consequence, researchers from disparate fields started to employ them in various applications $[15,16]$. The greater part of this application exploits the zonotope facility in defining robust approximations.

Zonotopes are also used to rigorously estimate the states of dynamical systems as an alternative to observers that optimize with respect to the best estimate, such as Kalman filters. One of the first works that use zonotopes for state-bounding observers is [17] and bounded disturbance in [18]. Similarly to reachability analysis, this work has been extended to nonlinear systems in $[19,20]$ and systems with uncertain parameters [21].

This paper is organised as follows, a description of the considered problem is first presented. Then, the optimal control problem for constrained uncertain systems is formulated. Its resolution procedure using zonotopic invariant sets with an interpolation step, is proposed. The efficiency of the used zonotopic invariant sets is then illustrated by two examples. Finally, the paper is concluded.

\section{Problem description}

The considered system is the following linear timevarying (LTV) system with polytopic uncertainty:

$$
\begin{aligned}
& x(k+1)=A(k) x(k)+B u(k) \\
& y(k)=C x(k)
\end{aligned}
$$

where $x(k)$ is the state of the plant, $u(k)$ is the control input and $y(k)$ is the plant output. We assume that:

$$
\begin{aligned}
& {[A(k), B(k)] \in \Omega,} \\
& \Omega=\operatorname{conv}\left\{\left[A_{1}, B_{1}\right],\left[A_{2}, B_{2}\right], \ldots,\left[A_{L}, B_{L}\right]\right\}
\end{aligned}
$$

where conv is the convex hull and Omega is a polytope, $\left[A_{j}, B_{j}\right]$ are vertices of the polytope such that:

$$
\left[A_{j}, B_{j}\right]=\sum_{j=1}^{L} \lambda_{j}\left[A_{j}, B_{j}\right], \sum_{j=1}^{L} \lambda_{j}=1,0 \leq \lambda_{j} \leq 1,
$$

The aim is this research is to find a state-feedback control law:

$$
u(k+i / k)=K x(k+i)
$$

that stabilizes (1) with the following performance cost:

$$
\begin{aligned}
& \min _{u(k+i / k)[A(k+i), B(k+i)] \in \Omega, i \geq 0} J_{\infty}(k) \\
& J_{\infty}(k)=\sum_{i=0}^{\infty}\left[\begin{array}{l}
x(k+i / k) \\
u(k+i / k)
\end{array}\right]^{T}\left[\begin{array}{cc}
\Theta & 0 \\
0 & R
\end{array}\right]\left[\begin{array}{l}
x(k+i / k) \\
u(k+i / k)
\end{array}\right]
\end{aligned}
$$

subject to :

$$
\begin{gathered}
\left|u_{h}(k+1 / k)\right| \leq u_{h, \text { max }}, h=1,2, \ldots, n_{u} \\
\left|y_{r}(k+1 / k)\right| \leq y_{r, \text { max }}, r=1,2, \ldots, n_{y}
\end{gathered}
$$

where $\Theta>0$ and $R>0$ are symmetric weighting matrices.

In [13] the authors describe the concept of an asymptotically stable invariant ellipsoid to develop a robust constrained MPC algorithm. This algorithm gives a sequence of explicit control laws corresponding to a sequence of asymptotically stable invariant ellipsoids constructed off-line one within another in state space. They solved, at each time step, the robust constrained MPC problem using Linear Matrix Inequalities (LMI). The obtained result is considered conservative due to invariant ellipsoids which are an approximation of the real invariant sets.

In [5] the authors describe polyhedral invariant sets with an off-line robust algorithm to stabilize uncertain systems. They are calculated off-line a sequence of state feedback control laws corresponding to a sequence of polyhedral invariant sets. At each sampling time, the smallest polyhedral invariant set that the currently measured state can be embedded is determined. The corresponding state feedback control law is then implemented to the process.

We intend to use this algorithms with zonotopic representation of the invariant sets followed by an interpolation step to get less conservative results.

\section{Robust MPC Algorithm}

In this section, an interpolation-based robust MPC algorithm for uncertain polytopic discrete-time systems using zonotopic invariant sets is presented. The nested zonotopic invariant sets and feedback gains are pre-computed off-line in first step, in order to reduce the on-line computational burdens. In second step, the real-time control law is calculated by linear interpolation between the feedback gains corresponding to the zonotopic invariant sets previously generated. The optimization problem solved at each time step is based on optimization of linear performance index and only a computationally low-demanding optimization problem is required to be solved on-line.

Definition 1: (Invariant sets)

An invariant set is a subset of the state space $\Omega \in R^{n}$, 
such that for all $x_{0} \in \Omega$, and all admissible input function $u: R \rightarrow U$, the solution to system (1) with $x(0)=x_{0}$ satisfies $x(t) \in \Omega$ for all $t \geq 0$.

Intuitively, the system remains trapped in the invariant for all future times [22].

One of the advantages of invariant sets, compared with iterative methods, is that they cover unbounded time horizon, without any extra cost. A second one is that they that have in general a compact representation. For example, an invariant ellipsoid is represented by a single nn matrix. Whereas, iterative methods produce a large number of sets, often with growing complexity. Each of these sets has to be taken into account in order to enclose all reachable states.

\subsection{Off-line Steps}

Step 1: Choose a state sequence $x_{i}, i \in\{1,2, \ldots, N\}$ and solve the following problem to obtain corresponding state feedback gains:

$$
K_{i}=Y_{i} Q_{i}^{-1}
$$

The states $x_{i}$ must be chosen such that the distance between $x_{i+1}$ and the origin is less than the distance between $x_{i}$ and the origin. Matrices $Y_{i}$ and $Q_{i}^{-1}$, for all $i=1,2, \ldots, N$ are solutions of the following problem:

$$
\min _{\gamma_{i}, Q_{i}, Y_{i}} \gamma_{i}
$$

subject to:

$$
\begin{aligned}
& {\left[\begin{array}{ll}
1 & x_{i}^{T} \\
x_{i} & Q_{i}
\end{array}\right] \geq 0} \\
& {\left[\begin{array}{llll}
Q_{i} & Q_{i} A_{j}^{T}+Y^{T} B_{j}^{T} & Q_{i} \Theta^{1 / 2} Y_{i}^{T} R^{1 / 2} \\
A_{j} Q_{i}+B_{j} Y_{i} & Q_{i} & 0 & 0 \\
\Theta^{1 / 2} Q_{i} & 0 & \gamma_{i} I & 0 \\
R^{1 / 2} Y_{i} & 0 & 0 & \gamma_{i} I
\end{array}\right] \geq 0} \\
& \forall j=1,2, \ldots, L \\
& {\left[\begin{array}{lc}
X & Y_{i} \\
Y_{i}^{T} & Q_{i}
\end{array}\right] \geq 0, X_{h h} \leq u_{h, \max }^{2}, h=1,2, \ldots, n_{u}} \\
& {\left[\begin{array}{lr}
S & C\left(A_{j} Q_{i}+B_{j} Y_{i}\right) \\
\left(A_{j} Q_{i}+B_{j} Y_{i}\right)^{T} C^{T} & Q_{i}
\end{array}\right] \geq 0, S_{r r} \leq y_{r, \max }^{2},} \\
& r=1,2, \ldots, n_{y}, \forall j=1,2, \ldots, L,
\end{aligned}
$$

Step 2: Given the state feedback gains:

$$
K_{i}=Y_{i} Q_{i}^{-1}, i \in\{1,2, \ldots, N\}
$$

from step 1. For each $K_{i}$, the corresponding polyhedral invariant sets defined by:

$$
S_{i}=\left\{x_{i} / M_{i} x_{i} \leq d_{i}\right\}
$$

are constructed by the following :

Step 2.1: Set $M_{i}=\left[C^{T},-C^{T}, K_{i}^{T},-K_{i}^{T}\right]^{T}, d_{i}=$ $\left[y_{\max }^{T}, y_{\min }^{T}, u_{\max }^{T}, u_{\min }^{T}\right]^{T}$ and $m=1$

Step 2.2: Select row $m$ from $\left(M_{i}, d_{i}\right)$ and check whether $M_{i, m}\left(A_{j}+B_{j} K_{i}\right) x \leq d_{i, m}$ is redundant with respect to the constraints defined by $\left(M_{i}, d_{i}\right)$ by solving the problem:

$$
\max _{x} W_{i, m, j}
$$

subject to

$$
W_{i, m, j}=M_{i, m}\left(A_{j}+B_{j} K_{i}\right) x-d_{i, m}, \quad M_{i} x \leq d_{i}
$$

Step 2.3: Let $m=m+1$ and return to Step 2.2. If $m$ is strictly larger than the number of rows in $\left(M_{i}, d_{i}\right)$ then terminate.

\subsection{On-line Step using polyhedral sets}

\subsubsection{Without interpolation}

At each sampling time, determine the smallest polyhedral invariant set $S_{i}=\left\{x_{i} / M_{i} x_{i} \leq d_{i}\right\}$ where $i=$ $1,2, \ldots, N-1$.

containing the measured states and implement the corresponding state feedback control law $u(k / k)=$ $K_{i} x(k / k)$ to the process.

\subsubsection{With 3-points interpolation}

At each sampling time, if the measured state lies between $S_{i}, S_{i+1}$ and $S_{i+2}, i=1,2, \ldots, N-1$ implement the interpolated gain obtained by :

$$
\mathrm{K}=\alpha_{1} K_{i-2}+\alpha_{2} K_{i-1}+\alpha_{3} K_{i}
$$

where $0<\alpha_{i}<1$, for all $i=1,2,3$ and $\sum_{i=1}^{3} \alpha_{i}=1$.

\subsection{On-line Step using zonotopic sets}

Zonotopes are convex polytopes that are centrally symmetric. Equivalently, a zonotope is a Minkowski sum of a finite set of line segments. A polytope is a zonotope if it can also be represented by so-called generators (G-representation).

Definition 2: (G-representation of a zonotope) Given a vector $c \in R^{n}$ and a set of vectors of $R^{n}, G=$ $\left\{g_{1}, \ldots, g_{m}\right\}, m \geq n$, a zonotope $Z$ of order $m$ is defined as following:

$$
Z=\left\{x \in R^{n}, x=c+\sum_{i=1}^{p} \gamma_{i} \cdot g_{i} ;-1 \leq \gamma_{i} \leq 1\right\}
$$

The vector $c$ is called the center of the zonotope $Z$. The vectors $g_{1}, \ldots, g_{m}$ are called generators of $Z$.

The order of zonotope is defined by the number of its generators ( $m$ in this case). In the case of $m<n$, its called degenerated zonotope.

This definition is equivalent with the definition of zonotopes by the Minskowski sum of a finite number of line segments defined by $g_{i} B^{1} . Z=\left(c ; g_{1}, g_{2}, \ldots, g_{m}\right)=$ $c \oplus g_{1} B^{1} \oplus \ldots \oplus g_{m} B^{1}$ Where $B^{n}$ is a unitary box in $R^{n}$, is a box composed by $\mathrm{n}$ unitary intervals. And $\oplus$ is the Minkowski sum.

Definition 3: (Minkowski sum) 
The Minkowski sum of two sets $\mathrm{X}$ and $\mathrm{Y}$ is defined by $X \oplus Y=\{x+y: x \in X, y \in Y\}$.

Definition 4: (Unitary interval)

The unitary interval is defined by $B^{n}=[-1,1]$.

Definition 5: (Box)

A box is an interval vector. An interval hull of a set $Z \subseteq R^{n}$, denoted by $\diamond Z$ is a box that satisfies $Z \subseteq \diamond Z$ Given a box $\diamond Z=\left(\left[a_{1}, b_{1}\right], \ldots,\left[a_{n}, b_{n}\right]\right)^{T} . \operatorname{mid}(\diamond Z)$, denotes its center and $\operatorname{diam}(\diamond Z)=\left(b_{1}-a_{1}, \ldots, b_{n}-a_{n}\right)^{T}$

Definition 6: (Unitary box)

A unitary box, denoted by $B^{n}$ is a box compound by $n$ unitary intervals.

Definition 7: (V-representation of a polytope)

Given $r$ vertices $v_{i} \in R^{n}, P=\operatorname{conv}\left\{v_{1}, \ldots, v_{r}\right\}$ is a convex polytope, where conv is the convex hull operator.

To obtain zonotopic sets from polyhedral ones, we have to perform the following three steps:

Step 1: Compute the vertices $v_{i} \in R^{n}(\mathrm{~V}-$ representation) of all $\mathrm{N}$ polytopes $S_{i}, i=1,, N$.

Step 2: Obtain the minimum and maximum values of each polytope $i$ :

$$
\begin{aligned}
& m_{\min }=\min \left(V_{i}^{1}, \ldots, V_{i}^{v}\right), \\
& m_{\max }=\max \left(V_{i}^{1}, \ldots, V_{i}^{v}\right) .
\end{aligned}
$$

where $V_{i}^{j}$ is the ith component of $v^{j}$ and $r$ is the number of the vertices of each polytope.

Step 3: Compute a G-representation of the ndimensional interval $\left[m_{\min }, m_{\max }\right]$ :

$$
\left[m_{\min }, m_{\max }\right]=\left\{x=c+\sum_{i=1}^{n} \gamma_{i} \cdot g_{i},-1 \leq \gamma_{i} \leq 1\right\}
$$

where :

$$
\begin{gathered}
c=0.5\left(m_{\min }+m_{\max }\right), \\
g_{i}^{(i)}=\left\{\begin{array}{l}
0.5\left(m_{\max }-m_{\min }\right), \text { if } i=j \\
0, \text { otherwise }
\end{array}\right.
\end{gathered}
$$

\subsubsection{Without interpolation}

At each sampling time, determine the smallest invariant zonotope

$Z=\left\{x \mid x=c+\sum_{i=1}^{p} \gamma_{i} \cdot g_{i},-1 \leq \gamma_{i} \leq 1\right\}, i=1,2, \ldots, N-1$

containing the measured states and implement the corresponding state feedback control law $u(k / k)=$ $K_{i} x(k / k)$ to the process.

\subsubsection{With 2-points interpolation}

At each sampling time, if the measured state lies between $Z_{i}$ and $Z_{i-1}$, implement the interpolated gain obtained by :

$$
K=\alpha K_{i}+(1-\alpha) K_{i+1}
$$

where $0<\alpha_{i}<1$, for all $i=1,2$, and $\sum_{i=1}^{2} \alpha_{i}=1$.

\subsection{3 with 3-points interpolation}

At each sampling time, if the measured state lies between $Z_{i}, Z_{i-1}$ and $Z_{i-2}$, implement the interpolated gain obtained by:

$$
K=\alpha_{1} K_{i-2}+\alpha_{2} K_{i-1}+\alpha_{3} K_{i}
$$

where $0<\alpha_{i}<1$,for all $i=1,2,3$, and $\sum_{i=1}^{3} \alpha_{i}=1$.

\section{Application}

In this section, we are going to present two examples allowing to implement the proposed approach. For both examples, the software Yalmip toolbox [23] in the MATLAB environment was used to compute the solution of the LMI minimization problem.

\subsection{Example 1}

Lets consider an uncertain non-isothermal CSTR [5] where the exothermic reaction $A B$ takes place. The reaction is irreversible and the rate of reaction is first order with respect to component A. A cooling coil is used to remove heat that is released in the exothermic reaction. The uncertain parameters are: the reaction rate constant $k_{0}$ and the heat of reaction $\Delta H_{r x n}$. The linearized model based on the component balance and the energy balance is given by the following state equations:

$$
\left\{\begin{array}{l}
x(t+1)=A(t) x(t)+B(t) u(t) \\
y(t)=C x(t)
\end{array}\right.
$$

where $x=\left[\begin{array}{l}C_{A} \\ T\end{array}\right]$ is the state vector $x(t)$ and $u=$ $\left[\begin{array}{l}C_{A, F} \\ F_{C}\end{array}\right]$ is the control input vector $u(t)$. Matrices are defined by:

$$
\begin{gathered}
A=\left(\begin{array}{cc}
-\frac{F}{V}-k_{0} e^{-E / R T_{s}} & -\frac{E}{R T_{s}^{2}} k_{0} e^{-E / R T_{s}} C_{A s} \\
\frac{-\Delta H_{r x n} k_{0} e^{-E / R T_{s}}}{\rho C_{p}} & -\frac{F}{V}-\frac{U A}{V \rho C_{p}} \\
-\Delta H_{r x n} \frac{E}{\rho C_{p} R T_{s}^{2}} k_{0} e^{-E / R T_{s}} C_{A s}
\end{array}\right) \\
B=\left[\begin{array}{cc}
\frac{F}{V} & 0 \\
0 & -2.098 \times 10^{5} \frac{T_{s}-365}{V \rho C_{p}}
\end{array}\right] \\
C=\left[\begin{array}{cc}
1 & 0 \\
0 & 1
\end{array}\right]
\end{gathered}
$$

Where $C_{A}$ is the concentration of $\mathrm{A}$ in the reactor, $C_{(A, F)}$ is the feed concentration of $A, T$ is the reactor temperature, and $F_{C}$ is the coolant flow. The operating parameters are: $F=1 \mathrm{~m}^{3} / \mathrm{min}, V=$ $1 \mathrm{~m}^{3}, k_{0}=10^{9}-10^{10} \mathrm{~min}^{-1}, \frac{E}{R}=8330.1 \mathrm{~K},-\Delta H_{r \times n}=$ $10^{7}-10^{8} \mathrm{cal} / \mathrm{kmol}, \rho=10^{6} \mathrm{~g} / \mathrm{m}^{3}, U A=5.34 \times$ $10^{6} \mathrm{cal} /(\mathrm{kmin})$ and $C_{p}=1 \mathrm{cal} /(\mathrm{gk})$. Let $\bar{C}_{A}=C_{A}-C_{A, e q}$, $\bar{T}_{A}=T-T_{e q}, \bar{C}_{A, F}=C_{A, F}-C_{A, F, e q}$ and $\overline{F_{C}}=F_{C}-F_{C, e q}$ where the subscript eq is used to denote the corresponding variable at equilibrium condition. By discretization, using a sampling time of $0.15 \mathrm{~min}$, the 
discrete-time model with $\left[\begin{array}{l}\bar{C}_{A}(k) \\ \bar{T}(k)\end{array}\right]$ as a state vector and $\left[\begin{array}{l}\bar{C}_{A, F}(k) \\ \bar{F}_{C}(k)\end{array}\right]$ as a control vector, is given as follows:

$$
\left\{\begin{array}{l}
x(k+1)=A(k) x(k)+B(k) u(k) \\
y(k)=C x(k)
\end{array}\right.
$$

where:

$$
\begin{aligned}
& A=\left[\begin{array}{cc}
0.85-0.0986 \alpha(k) & -0.0014 \alpha(k) \\
0.9864 \alpha(k) \beta(k) & 0.0487+0.01403 \alpha(k) \beta(k)
\end{array}\right] \\
& B=\left[\begin{array}{cc}
0.15 & 0 \\
0 & -0.912
\end{array}\right], \quad C=\left[\begin{array}{cc}
1 & 0 \\
0 & 1
\end{array}\right]
\end{aligned}
$$

where: $1 \leq \alpha(k)=k_{0} / 10^{9} \leq 10$ and $1 \leq \beta(k)=$ $-\Delta H_{r x n} / 10^{7} \leq 10$.

The two parameters $(k)$ and $(k)$ are independent of each other. Then, we consider the following polytopic uncertain model with four vertices:

$$
\Omega=\operatorname{Co}\left\{\left[\begin{array}{ll}
0.751 & -0.0014 \\
0.986 & 0.063 \\
0.751 & -0.0014 \\
0.986 & 0.063
\end{array}\right],\left[\begin{array}{ll}
0.751 & -0.0014 \\
9.864 & 0.189 \\
0.751 & -0.0014 \\
9.864 & 0.189
\end{array}\right],\right.
$$

The objective is to regulate the concentration $\bar{C}_{A}$ and the reactor temperature $\bar{T}$ to the origin by manipulating $\bar{C}_{A, F}$ and $\bar{F}_{C}$, respectively. These variables are constrained by: $\left|\bar{C}_{A, F}\right| \leq 0.5 \mathrm{kmol} / \mathrm{m}^{3}$, and $\left|\bar{F}_{C}\right| \leq$ $1.5 \mathrm{~m}^{3} / \mathrm{min}$.

The cost function is given by (5) with $\Theta=I$ and $R=0.1 I$.

Lets choose a sequence of states:

$$
x_{i}=\left\{\begin{array}{c}
(0.0525,0.0525),(0.0475,0.0475) \\
(0.0425,0.0425),(0.0375,0.0375) \\
(0.0325,0.0325),(0.0275,0.0275)
\end{array}\right\}
$$

This sequence is used to calculate six off-line feedback gains $K_{i}, i=1,2, \ldots, 6$. The regulated output (the concentration of $\mathrm{A}$ and the reactor temperature), when $\alpha(k)$ and $\beta(k)$ are randomly time-varying between $10^{9} \leq \alpha(k)=10^{10}$ and $10^{7} \leq \beta(k)=\Delta H_{r \times n} \leq 10^{8}$.

The obtained zonotopes are defined by:

$$
c_{i}=\{2.98,3.17,-1.31,1.31,-3.17,-2.98\},
$$

Where $c_{i}$ is the center of the zonotope $Z_{i}, i=1,2, . ., 6$. The generators matrices are defined by:

$$
g_{i}=\left(\begin{array}{cccccc}
3.07 & 0 & 0 & 0 & 0 & 0 \\
0 & 3.26 & 0 & 0 & 0 & 0 \\
0 & 0 & 1.29 & 0 & 0 & 0 \\
0 & 0 & 0 & 1.29 & 0 & 0 \\
0 & 0 & 0 & 0 & 3.26 & 0 \\
0 & 0 & 0 & 0 & 0 & 3.07
\end{array}\right)
$$

for all $i=1,2, . ., 6$.

The regulated outputs are shown respectively in Figure 1 and Figure 2. It is seen that the considered zonotopic sets give less conservative results and better system performance as compared to the approach using polyhedral ones.

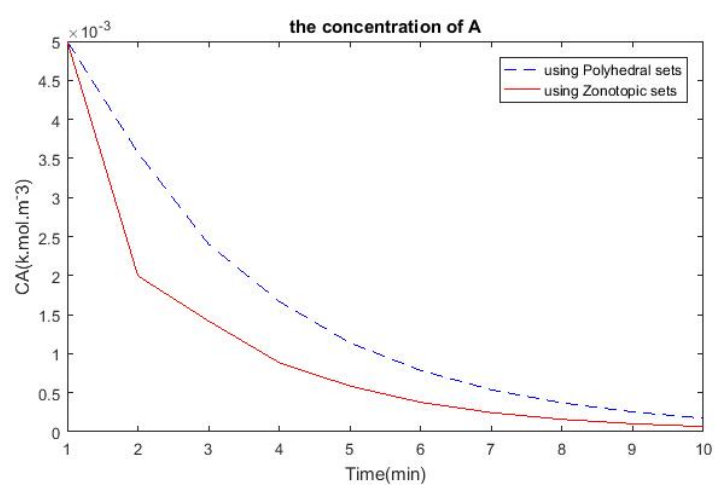

Figure 1: The concentration of $\mathrm{A}$ in the reactor of the

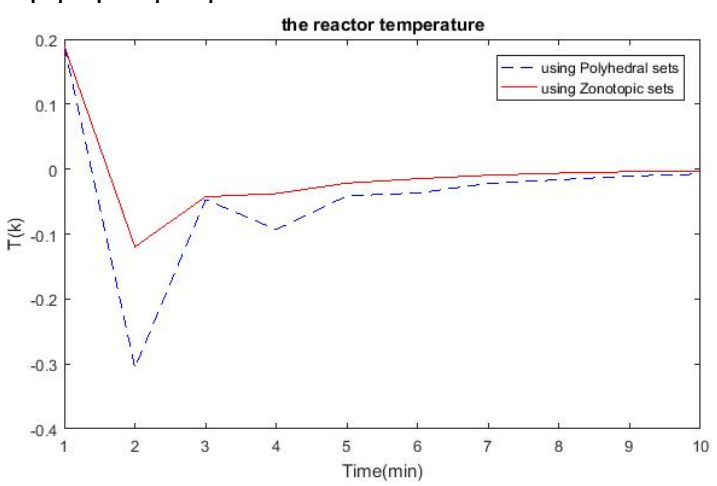

Figure 2: The reactor temperature of the regulated output Figure 3 and Figure 4 respectively, it is seen that the considered interpolation using three zonotopic sets, give less conservative results as compared to the approach with interpolation of polyhedral sets.

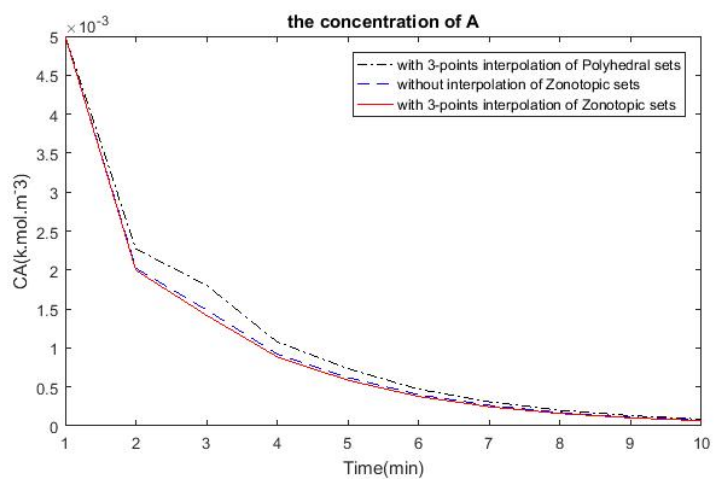

Figure 3: The concentration of $\mathrm{A}$ in the reactor of the regulated output

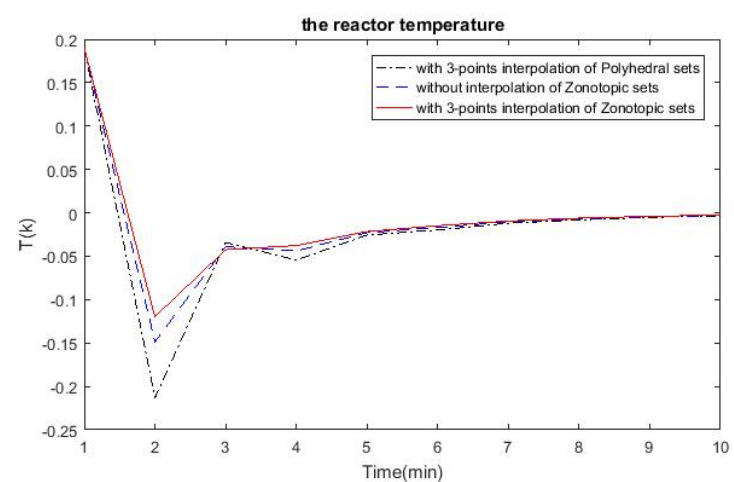

Figure 4: The reactor temperature of the regulated output 


\subsection{Example 2}

We consider the angular positioning system [4]. It consists of an electric motor driving a rotating antenna so that it always points in the direction of a moving object. The motion of the antenna can be described by the following discrete time-equation:

$$
\left\{\begin{array}{c}
{\left[\begin{array}{c}
\theta(k+1) \\
\dot{\theta}(k+1)
\end{array}\right]=\left[\begin{array}{cc}
1 & 0.1 \\
0 & 1-0.1 \alpha(k)
\end{array}\right]\left[\begin{array}{c}
\theta(k) \\
\dot{\theta}(k)
\end{array}\right]} \\
+\left[\begin{array}{l}
0 \\
0.0787
\end{array}\right] u(k) \\
y(k)=\left[\begin{array}{ll}
1 & 0
\end{array}\right]\left[\begin{array}{l}
\theta(k) \\
\dot{\theta}(k)
\end{array}\right]
\end{array}\right.
$$

where $\theta(k)$ is the angular position of the antenna, $\dot{\theta}(k)$ is the angular velocity and $u(k)$ is the input voltage of the motor. It is assumed that the uncertain parameter is arbitrarily time-varying : $0.1 \alpha(k) 10$.

Let $\bar{\theta}=\theta-\theta_{e q}, \overline{\dot{\theta}}=\dot{\theta}-\dot{\dot{\theta}}$ and $\bar{u}=u-u_{e q}$ where the subscript $e q$ denotes the corresponding variable at equilibrium condition. The obtained system can be written as follows:

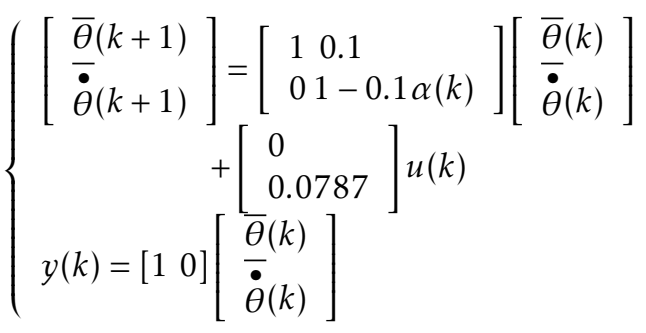
ture:

The system (36) has the following polytopic struc-

$$
A(k) \in \operatorname{conv}\left\{\left[\begin{array}{ll}
1 & 0.1 \\
0 & 0.9
\end{array}\right],\left[\begin{array}{ll}
1 & 0.1 \\
0 & 0
\end{array}\right]\right\}
$$

The input constraint is:

$$
\overline{|\bar{u}(k)|} \leq 2 \text { volts }
$$

The weighting matrices $\Theta$ and $R$ are given by:

$$
\Theta=\left[\begin{array}{ll}
1 & 0 \\
0 & 0
\end{array}\right] \text { and } R=0.00002 I
$$

Lets choose the following sequence of seven states:

$$
x_{i}=\left\{\begin{array}{l}
(0.35,0.35),(0.3,0.3), \\
(0.25,0.25),(0.02,0.02), \\
(0.15,0.15),(0.1,0.1),(0.05,0.05)
\end{array}\right\}
$$

This sequence is used to calculate seven state feedback gains $K_{i}$ corresponding to seven polyhedral invariant sets. The obtained zonotopes are defined by their centers:

$$
c_{i}=\left\{\begin{array}{l}
1.52,-0.08,-0.21,0.21, \\
0.08,-1.52,0.21
\end{array}\right\} i=1,2, \ldots, 7 .
$$

The zonotope generators are given by:

$$
g_{i}=\left(\begin{array}{ccccccc}
0.82 & 0 & 0 & 0 & 0 & 0 & 0 \\
0 & 3.87 & 0 & 0 & 0 & 0 & 0 \\
0 & 0 & 0.70 & 0 & 0 & 0 & 0 \\
0 & 0 & 0 & 3.38 & 0 & 0 & 0 \\
0 & 0 & 0 & 0 & 0.59 & 0 & 0 \\
0 & 0 & 0 & 0 & 0 & 2.87 & 0 \\
0 & 0 & 0 & 0 & 0 & 0 & 0.47
\end{array}\right)
$$

for all $i=1,2, \ldots, 7$.

Figure 5 and Figure 6 represent closed-loop responses of the system when $\alpha(k)$ is randomly time-varying between $0.1 \leq \alpha(k) \leq 10$.

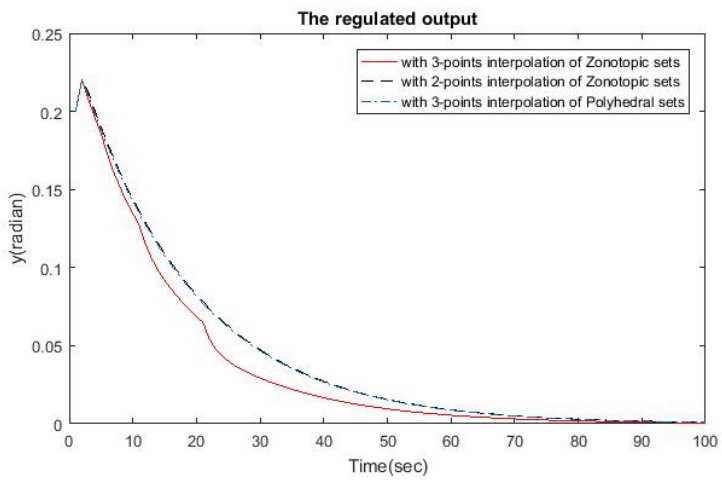

Figure 5: The regulated output

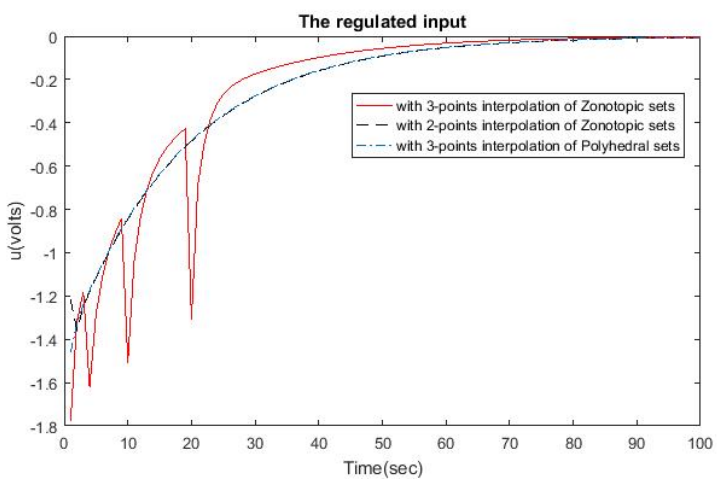

Figure 6: The control input

We can observe that by the considered approach with zonotopic sets using three points interpolation especially the one with three control gains, we obtain better control performances as compared to the approach with interpolation of polyhedral sets.

\section{Conclusion}

In this paper, we have presented an input feedback robust model predictive control of polytopic uncertain discrete-time systems. The proposed algorithm used an off-line optimal control optimization problems solution to determine a sequence of feedback gains. A sequence of nested zonotopic invariant sets associated with pre-computed feedback gains are constructed. At each control iteration, the smallest invariant containing the measured states is identified, and the corresponding feedback gain is implemented. In 
addition, an interpolation step to the obtained control laws based on polyhedral and zonotopic invariant sets respectively was employed. The proposed approach applied on examples showed that the control performance using zonotopic invariant sets followed by an interpolation of the nested zonotopes is better than the one using polyhedral invariant sets.

\section{References}

1. S. Kheawhom and P. Bumroongsri. Interpolation-based robust constrained model predictive output feedback control, in Conference on Control and Automation. June 16-19. Palermo, Italy, 2014.

1. B. Ding, Y. Xi, M. T. Cychowski and T.O.Mahony. Improving off-line approach to robust MPC based-on nominal performance cost, Automatica, vol. 43, No. 1, pp. 158163, 2007.

2. X. Liu, S. Feng and M. Ma, Robust MPC for the constrained system with polytopic uncertainty. International Journal of Systems Science, vol. 43, No. 2, pp. 248258, 2012 ..

3. F. Borelli. Constrained optimal control of linear and hybrid systems, vol 290 of Lecture Notes in Control and Information Sciences, Springer, 2010.

4. M. H. Nehrir, C. Wang, Modeling and Control of Fuel Cells: Distributed Generation Applications, Wiley-IEEE Press, 2009.

5. B. Pornchai and K. Soorathep. An off-line robust MPC algorithm for uncertain polytopic discrete-time systems using polyhedral invariant sets, Journal of Process Control, vol.22, No. 5, pp. 975-983, 2012.

6. F. Blanchini and S. Miani.Set-theoretic methods in control. Systems and Control, Foundations and Applications, 2008.

7. A. Bemporad. M. Morari, V. Dua. and E. N. Pistikopoulos. The explicit linear quadratic regulator for constrained systems, Automatica, vol. 38, pp. 3-20, 2002.

8. W. Bey, Z. Kardous and N. Benhadj Braiek. Stabilization of Constrained uncertain systems by Multi-Parametric Optimization,International Journal of Automation and Control(IJAAC), vol. 10, n. 4, pp. 407-416, Inderscience Enterprises Ltd, 2016.

9. A. B. Kurzhanskii and I. Vlyi. Ellipsoidal calculus for estimation and control, Burlhauser, Boston, Massachusets, 1997.

10. A. C. Brooms, B. Kouvaritakis, and Y. I. Lee. Constrained MPC for uncertain linear systems with ellipsoidal target sets, Systems and Control Letters, vol. 44, No. 3, pp. 157166, 2011.
11. A. Matthias, S. Olaf and B. Martin. Computing reachable sets of hybrid systems using a combination of zonotopes and polytopes, Nonlinear Analysis: Hybrid Systems, vol.4, No. 2,pp. 233-249, 2010.

12. A. Casavola, D. Angeli, G. Franze and E.Mosca. An ellipsoidal off-line MPC scheme for uncertain polytopic discretetime systems, Automatica, vol. 44, No. 12, pp. 31133119, 2008.

13. Z. Wan and M. V. Kothare, An efficient off-line formulation of robust model predictive control using linear matrix inequalities. Automatica, vol. 39, No. 5, pp. 837846, 2003.

14. A. Ingimundarson, J. M. Bravo, V. Puig, T. Alamo and P. Guerra. Robust fault detection using zonotope-based setmembership consistency, International journal of adaptive control and signal processing, vol. 23, No. 4, pp. 311330, 2008.

15. M. Althoff, O. Stursberg and M. Buss. Computing reachable sets of hybrid systems using a combination of zonotopes and polytopes, Nonlinear Analysis: Hybrid Systems, vol. 4, No. 2, pp. 233249, 2010.

16. J. Blesa, V. Puig and J. Saludes. Identification for passive robust fault detection using zonotope-based set-membership approaches, International Journal of Adaptive Control and Signal Processing, vol.25, No.9, pp. 788-812, 2011.

17. C. Combastel. A state bounding observer based on zonotopes, In Proceeding of the European Control Conference, 2003.

18. F. Stoican, S. Olaru, J. A. De Don and M. M. Seron. Zonotopic ultimate bounds for linear systems with bounded disturbances, In Proceedings of the 18th IFAC World Congress, Milano, Italy, pp. 92249229, 2011.

19. T. Alamo, J. M. Bravo and E. F. Camacho. Guaranteed state estimation by zonotopes, In Proceeding of the 42nd IEEE Conference on Decision and Control, pp. 58315836, 2003.

20. C. Combastel. A state bounding observer for uncertain nonlinear continuous-time systems based on zonotopes, In Proceeding of the 44th IEEE Conference on Decision and Control, and the European Control Conference, ECC, pp.72287234, 2005.

21. V. T. H. Le, C. Stoica, T. Alamo, E. F. Camacho and D. Dumur. Zonotopic guaranteed state estimation for uncertain systems. Automatica, vol. 49, No. 11, pp.4183424, 2013.

22. I. Ben Makhlouf, P. Hansch and S. Kowalewski. Comparison of Reachability Methods for Uncertain Linear TimeInvariant Systems, European Control Conference (ECC) July 17-19 Zrich, Switzerland, 2013.

23. J. Lofberg, Yalmip: A toolbox for modeling and optimization in matlab, in Proc. IEEE international symposium on computer aided control systems design, pp. 284289, 2004. 